\title{
Papers
}

\section{A two year prospective study to compare culture and polymerase chain reaction amplification for the detection and diagnosis of Lyme borreliosis}

Department of

Pathology, Room 2242, Building 110, Loyola

University Medical Center and Hines

Veterans'

Administration

Hospital, 2160 South

First Avenue,

Maywood, IL 60153,

USA

M M Picken

Section of Infectious

Disease,

Rush-Presbyterian-St

Luke's Medical Center, 600 South Paulina

Avenue, Chicago, IL

60612, USA

R N Picken

D Han

Y Cheng

Department of

Infectious Diseases,

University Medical

Centre, Japljeva 2, 1525

Ljubljana, Slovenia

J Cimperman

V Maraspin

$S$ Lotric-Furlan

F Strle

Institute of

Microbiology,

University of

Ljubljana, Zaloska 4,

1105 Ljubljana,

Slovenia

E Ruzic-Sabljic

Correspondence to

Dr M M Picken.

Accepted for publication

24 April 1997

M M Picken, R N Picken, D Han, Y Cheng, E Ruzic-Sabljic, J Cimperman, V Maraspin, S Lotric-Furlan, F Strle

\section{Abstract}

Aim-To compare polymerase chain reaction (PCR) amplification of borrelial DNA and culture isolation of spirochaetes for the diagnosis of Lyme borreliosis by direct detection of Borrelia burgdorferi sensu lato in patients with erythema migrans and acrodermatitis chronica atrophicans lesions.

Methods-Skin biopsy specimens from erythema migrans and acrodermatitis chronica atrophicans lesions were subdivided and tested by PCR amplification assay and culture using two artificial growth media, Barbour-Stoenner-Kelly II (BSK II) and modified Kelly-Pettenkofer (MKP). Five classes of lesions were studied: typical erythema migrans, spontaneously resolved erythema migrans, atypical/partially treated erythema migrans, typical acrodermatitis chronica atrophicans, and atypical/partially treated acrodermatitis chronica atrophicans.

Results-For both erythema migrans and acrodermatitis chronica atrophicans lesions, the most sensitive detection method was MKP culture. PCR was less sensitive than MKP culture, but more sensitive than BSK II culture. Results for 758 typical erythema migrans specimens showed positivity rates of $36 \%$ for MKP, $25 \%$ for PCR, and $24 \%$ for BSK II. Differences were statistically significant. The overall positivity rate for all three methods combined was $54 \%$, but few specimens (6\%) were positive by all three methods. Examination of multiple erythema migrans lesions from the same patient increased the diagnostic yield. These findings, and similar results for acrodermatitis chronica atrophicans lesions, suggest that the distribution of spirochaetes in skin biopsies is not homogeneous.

Conclusions-Although possessing the potential to provide a rapid diagnosis, PCR is not more sensitive than culture for the direct detection of borrelia. Spirochaetes appear to be unevenly distributed throughout biopsy specimens, suggesting that diagnosis of Lyme borreliosis by direct detection of the causative agent in $\vec{\circ}$ skin lesions is vulnerable to sample bias. (F Clin Pathol: Mol Pathol 1997;50:186-193)

Keywords: culture isolation; polymerase chain reaction; Borrelia burgdorferi sensu lato

Lyme borreliosis is the most common. arthropod-borne human infection in Europe and North America. ${ }^{1{ }^{2}}$ It is a multisystem disorder, transmitted by ticks of the Ixodes rici- $\bar{O}$ nus complex, ${ }^{3}$ and caused by spirochaetes of $\stackrel{0}{\circ}$ the general taxon Borrelia burgdorferi sensu $\stackrel{\mathbb{2}}{2}$ lato. ${ }^{4}$ In Europe, at least three species are $\overrightarrow{0}$ known to be associated with human disease: $B 3$ burgdorferi sensu stricto, $B$ garinii, and $\supsetneq$ $B$ afzelii. ${ }^{5}$ The clinical manifestations are multifarious and vary with disease progression. Early disease is often characterised by the appearance of an expanding erythematous skin 3 . rash, erythema migrans, at the site of the tick bite $^{78}$ and, for many patients, the instigation of appropriate antibiotic therapy at this stage is 응 effective in eradicating the infection. ${ }^{9} 10$ Early $>$ diagnosis and treatment is important as, if untreated, the disease may progress to involve $N$ other organ systems such as the nervous system, heart, and joints. ${ }^{8}$ Treatment of early disseminated infection is less effective than $\omega$ therapy of erythema migrans and chronic disease manifestations such as chronic Lyme arthritis, neurological disease, and the atrophic $\stackrel{\oplus}{\Phi}$ skin condition acrodermatitis chronica atrophicans ${ }^{7}$ respond very indifferently to antimicrobial therapy.

In the case of patients from endemic regions $\frac{\Omega}{\square}$ presenting with typical erythema migrans and a $\frac{}{\sigma}$ firm recollection of a tick bite, a straightforward diagnosis of Lyme borreliosis usually can be made on clinical grounds alone. However, in a substantial number of cases, erythema $\frac{\overline{0}}{\partial}$ migrans may not occur, may be atypical, or both the tick bite and erythema migrans rash may go unnoticed by the patient. ${ }^{7}$ In such cases, confirmation by laboratory methods is desirable. Hitherto, serological testing has been the most widely used diagnostic method. However, serological tests have suffered from, and 
continue to suffer from, a number of problems. For example, many patients with early Lyme borreliosis are seronegative at presentation ${ }^{11}$ or antibody responses may be abrogated by early antibiotic treatment. ${ }^{12}$ False positive results can arise as a consequence of cross-reactivity with epitopes present on other microorganisms, ${ }^{13}$ and antibody titres may persist in patients who were previously infected and successfully treated, ${ }^{14}$ or after asymptomatic infection. Finally, there is a high degree of polymorphism among important immunogenic proteins of borrelial species and subtypes associated with human disease. ${ }^{15}$ Although it has been shown that confirmation of serological results by immunoblotting can increase the specificity of these tests, ${ }^{16}$ direct detection of spirochaetes continues to inspire the most confidence among clinicians.

Traditional clinical pathology methods used for the direct detection of spirochaetes have included culture in Barbour-Stoenner-Kelly II $(B S K ~ I I){ }^{17}$ and modified Kelly-Pettenkofer $(\mathrm{MKP})^{18}$ artificial media and staining of organisms in tissue sections. Inherent drawbacks of the former technique include difficulties in ensuring the consistency of prepared media and the extremely slow growth rate of some isolates. The latter technique has found a limited application because of the low frequency of positive sections. For these reasons, new methods of direct detection have been sought eagerly and quickly seized upon. Over the past decade, techniques of in vitro amplification of DNA molecules (as exemplified by polymerase chain reaction (PCR)) have been shown to provide both high sensitivity and specificity and have, therefore, been exploited widely for the direct detection of microorganisms. ${ }^{19}$ PCR has been used to detect borrelia in a variety of human and animal specimens, using primers and probes directed against a variety of target DNA sequences. ${ }^{20}$ Previously, we developed a conventional PCR assay for $B$ burgdorferi sensu lato based on conserved sequences and, therefore, able to detect a wide range of human borrelia isolates. ${ }^{21}$ The aim of the present study was to compare this PCR assay with culture for the detection of disease in patients with suspected Lyme borreliosis.

\section{Methods}

PATIENT SPECIMENS AND CULTURE

Five groups of patients were studied: (a) patients with typical erythema migrans lesions of recent onset (including multiple erythema migrans lesions); (b) patients with erythema migrans lesions that had resolved without antibiotic treatment (erythema migrans status post); (c) patients with atypical erythema migrans lesions and/or partial antibiotic therapy; (d) patients with classic acrodermatitis chronica atrophicans lesions who had not received antibiotic treatment specifically for this condition; and (e) patients with atypical acrodermatitis chronica atrophicans lesions and/or partial antibiotic treatment for acrodermatitis chronica atrophicans.

Criteria for the classification of typical erythema migrans lesions were those recom- mended by the Centers for Disease Control and Prevention (Atlanta, Georgia, USA). ${ }^{22}$ Atypical erythema migrans lesions comprised rashes that did not fulfill CDC criteria; examples include rashes that developed shortly after a tick bite, were smaller than $5 \mathrm{~cm}$ in diameter, and/or were non-expanding. Acrodermatitis chronica atrophicans lesions were classified as typical when patients reported longlasting, slowly expanding skin changes on distal parts of limbs, or when red or bluish-red oedematous or atrophic skin was found at these locations on examination. Additional requisite criteria included histological findings supporting the diagnosis of acrodermatitis chronica atrophicans, and the presence of serum IgG antibody titres against $B$ burgdorferi sensu lato. Acrodermatitis chronica atrophicans lesions were classified as atypical if one or more of these features were absent or inconclusive.

All patients included in the study presented to the Department of Infectious Diseases, University Medical Centre (UMC), Ljubljana, Slovenia between November 1992 and December 1994. The UMC is a national Lyme borreliosis reference centre, receiving patients with early disease from the surrounding area and patients with disseminated disease from the greater part of Slovenia.

For patients with extant or resolved skin manifestations, elliptical skin biopsy specimens $(8 \times 5 \times 3 \mathrm{~mm})$ were excised as described previously ${ }^{23}$ and divided into four equal segments. These were placed in: (a) modified BSK II medium containing rifampicin $(40 \mu \mathrm{g} /$ $\mathrm{ml}$ ) and ciprofloxacin $(4 \mu \mathrm{g} / \mathrm{ml})$; (b) $M K P$ medium containing co-trimoxazole $(30 \mu \mathrm{g} / \mathrm{ml})$; (c) tissue digestion buffer $(25 \mathrm{mM}$ Tris $\mathrm{HCl}$ pH 8.0, $25 \mathrm{mM}$ EDTA pH 8.0, 1.2\% vol/vol Triton X-100) for PCR processing; and (d) frozen storage at $-70^{\circ} \mathrm{C}$.

BSK II cultures and PCR samples were shipped to Chicago by express mail. Cultures were then diluted into fresh BSK II medium and PCR specimens were processed as described below. MKP and BSK II cultures were incubated at $33^{\circ} \mathrm{C}$ and checked weekly for the growth of spirochaetes by dark-field microscopy.

PRE AND POST-PCR LABORATORY CONFIGURATION To obviate the possibility of amplicon contamination, pre and post-PCR manipulations were carried out in separate institutions. In the prePCR stages, reagents and clinical samples were prepared in separate, adjacent laboratories in the same institution; each laboratory had its own set of equipment and there was no transfer of equipment between laboratories. PCR reactions were set up in a dedicated biological containment cabinet, using another separate set of equipment. The usual standard precautions against contamination of PCR reactions (aerosol barrier pipette tips, single use disposable plasticware, etc) were used. After addition of the sample, PCR reactions were sealed and transported on ice to a second institution $(-20$ minute duration), where they underwent thermocycling and amplicon analysis by agarose gel electrophoresis and Southern hybridisation. 


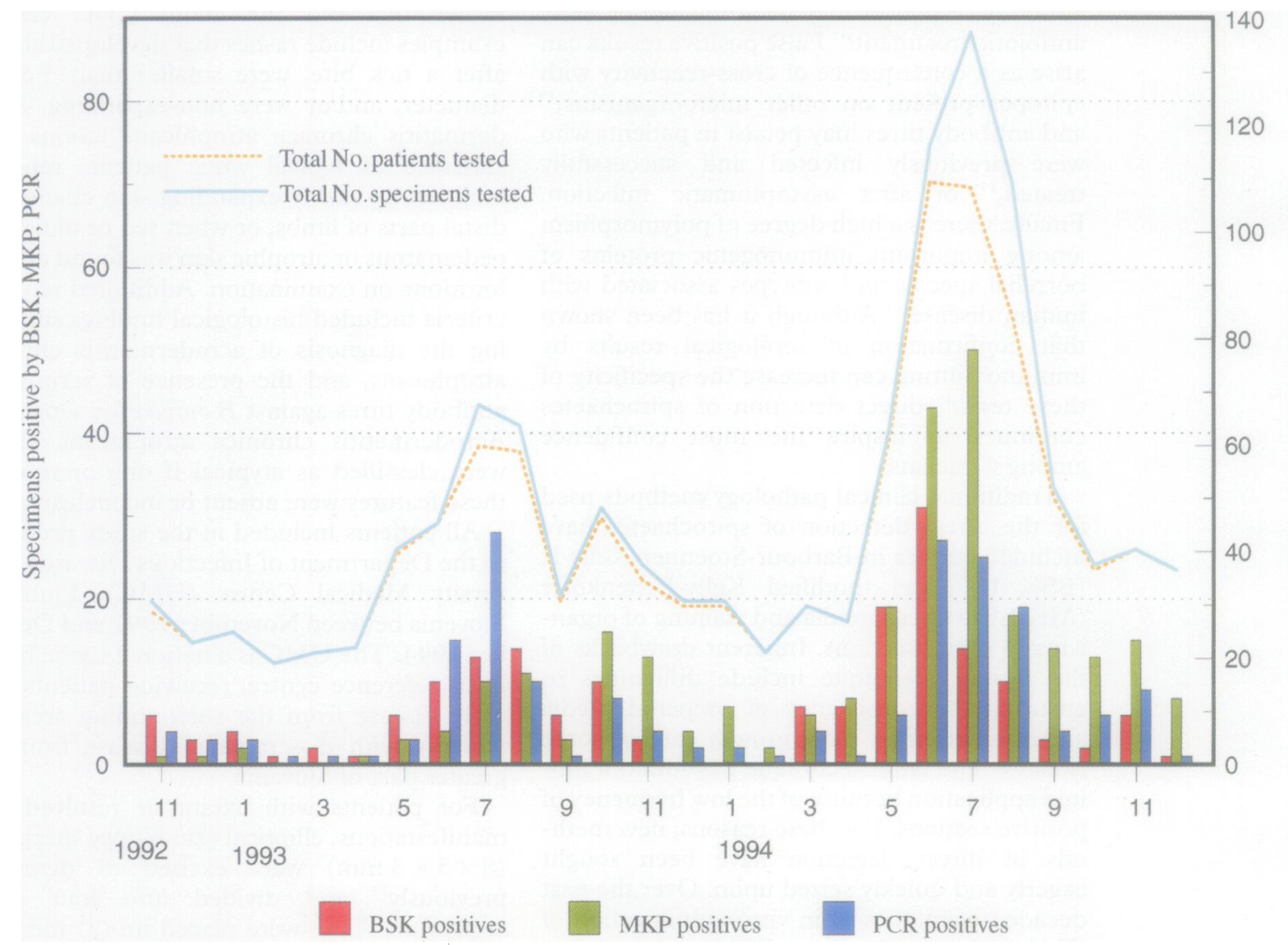

Figure 1 Numbers of typical erythema migrans lesions positive for B burgdorferi sensu lato by culture in BSK II medium, culture in MKP medium, or PCR amplification assay, compared with the total numbers of patients and patient specimens tested for each month of the study from November 1992 until December 1994.

SAMPLE PREPARATION FOR PCR

Skin specimens in tissue digestion buffer were brought into solution by the addition of proteinase $\mathrm{K}$, to a final concentration of $1.5 \mathrm{mg} / \mathrm{ml}$, and incubation overnight at $55^{\circ} \mathrm{C}$. The first 62 skin specimens were then processed for PCR by sequential extraction with phenol, phenol/ chloroform, and chloroform. Nucleic acids were precipitated with ethanol, dried in vacuuo, and dissolved in TE buffer $(10 \mathrm{mM}$ Tris $\mathrm{HCl}, 1 \mathrm{mM}$ EDTA, $\mathrm{pH} 8.0$ ). However, this technique was found not to be completely effective in removing PCR inhibitors from all skin specimens. Also, it involved many "open tube" manipulations, which carry an attendant risk of cross-contamination. For these reasons, subsequent skin specimens were prepared using the sample preparation protocol described by Boom et al. ${ }^{24}$ Skin specimens were brought into solution as described previously, followed by the addition of $1 \mathrm{ml}$ of $\mathrm{L} 2$ buffer. $^{24}$ Subsequent steps were performed as described by Boom et al. ${ }^{24}$

To test for the presence of PCR inhibitors in preparations of skin specimens, ${ }^{\prime} \mathrm{PCR}$ reactions were set up, transported to the amplicon analysis laboratory, and spiked with $10 \mathrm{pg}$ of purified $B$ burgdorferi sensu stricto strain B31 DNA. In the absence of inhibitors, this amount of template DNA produces an amplification product that is easily visible by agarose gel electrophoresis and ethidium bromide stain- $O$ ing. Specimens prepared using the phenol

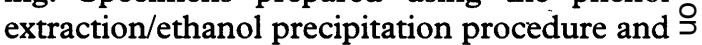
found to contain inhibitors were repurified $\frac{D}{O}$ using the "powdered glass" technique of Vogelstein and Gillespie. ${ }^{25}$ Samples prepared 0 according to Boom et $a l^{4}$ were also tested for N the presence of inhibitors by spiking with puri- N fied strain B31 DNA. During the testing of more than 200 skin specimens no inhibition 6 was detected and, therefore, the routine $\overparen{\Phi}$ "spike" testing of samples prepared by this ? procedure was discontinued.

Skin biopsy specimens from patients without $\frac{\vec{d}}{\mathrm{D}}$ Lyme symptomatology were obtained from the $\stackrel{?}{?}$ Department of Pathology, Loyola University $\unrhd$ Medical Center and co-processsed as controls.

PCR ASSAY

This was performed using the conventional flagellin gene PCR assay described previously. ${ }^{21}{ }^{26}$ Forty five cycles of amplification were used. Amplification products were detected by agarose gel electrophoresis, Southern hybridisation, and probing with $a{ }^{32} \mathrm{P}$ end labelled oligonucleotide probe as described previously. ${ }^{21}$ It has been shown that the sensitivity of this assay is $\sim 10$ spirochaetes. ${ }^{26}$ 
Table 1 Direct detection of Borrelia burgdorferi sensu lato in 758 skin biopsy specimens from classic erythema migrans lesions using culture in BSK II medium, culture in $M K P$ medium, and PCR amplification assay

\begin{tabular}{lllc}
\hline$B S K$ & $M K P$ & $P C R$ & Number \\
\hline+ & + & + & 47 \\
+ & + & - & 54 \\
+ & - & + & 34 \\
- & + & + & 50 \\
- & + & - & 121 \\
- & - & + & 58 \\
+ & - & - & 44 \\
- & - & - & 350 \\
\hline
\end{tabular}

Table 2 Direct detection of Borrelia burgdorferi sensu lato in skin biopsy specimens from multiple erythema migrans lesions of 14 patients using culture in BSK II medium, culture in MKP medium, and PCR amplification assay

\begin{tabular}{|c|c|c|c|c|}
\hline $\begin{array}{l}\text { Patient } \\
\text { number }\end{array}$ & Sample & $B S K$ & $M K P$ & $P C R$ \\
\hline \multirow[t]{2}{*}{1} & A & - & - & - \\
\hline & B & + & - & + \\
\hline \multirow[t]{2}{*}{2} & A & + & - & - \\
\hline & B & + & - & + \\
\hline \multirow[t]{2}{*}{3} & A & - & + & - \\
\hline & B & - & - & - \\
\hline \multirow[t]{2}{*}{4} & A & - & - & + \\
\hline & B & - & - & - \\
\hline \multirow[t]{2}{*}{5} & A & - & - & + \\
\hline & B & - & - & + \\
\hline \multirow[t]{2}{*}{6} & A & - & - & + \\
\hline & B & - & - & - \\
\hline \multirow[t]{2}{*}{7} & A & + & - & - \\
\hline & B & + & - & + \\
\hline \multirow[t]{2}{*}{8} & A & - & + & + \\
\hline & B & - & + & + \\
\hline \multirow[t]{2}{*}{9} & A & + & - & + \\
\hline & B & + & - & + \\
\hline \multirow[t]{2}{*}{10} & A & - & - & - \\
\hline & B & - & - & + \\
\hline \multirow[t]{2}{*}{11} & A & + & + & - \\
\hline & B & - & + & - \\
\hline \multirow[t]{2}{*}{12} & A & + & - & - \\
\hline & B & - & - & - \\
\hline \multirow[t]{2}{*}{13} & A & - & - & - \\
\hline & B & - & + & + \\
\hline \multirow[t]{2}{*}{14} & A & + & - & - \\
\hline & B & - & - & + \\
\hline
\end{tabular}

STATISTICAL ANALYSIS

Differences in the qualitative data were analysed using Yates corrected $\chi^{2}$ or Fischer's exact test; $\mathrm{p}$ values were two tailed.

\section{Results}

In the preparation of patient specimens for the PCR assay, preliminary results demonstrated no detectable difference between the two sample preparation protocols with regard to numbers of positive and negative samples. ${ }^{27}$ Therefore, results have been combined for presentation in fig 1 and tables 1-4. Control specimens, co-processed to check for cross- or carryover contamination, and control PCR reactions containing only distilled water, were negative.

\section{CLASSIC LESIONS}

The total numbers of positives obtained by BSK II culture, MKP culture, and PCR assay relative to the total number of patients and erythema migrans specimens tested during each month of the two year study are shown in fig 1 . As expected, the bulk of positive biopsies were obtained during the summer months of May, June, July, and August.

An immediate and surprising finding of these studies was that relatively few biopsy
Table 3 Direct detection of Borrelia burgdorferi sensu lato in 120 skin biopsy specimens from spontaneously resolved erythema migrans lesions (EM status post) using culture in $B S K$ II medium, culture in MKP medium, and PCR amplification assay

\begin{tabular}{llll}
\hline BSK & $M K P$ & $P C R$ & Number \\
\hline+ & + & + & 2 \\
+ & + & - & 0 \\
+ & - & + & 1 \\
- & + & + & 2 \\
- & + & - & 1 \\
- & - & + & 3 \\
+ & - & - & 1 \\
- & - & - & 110 \\
\hline
\end{tabular}

Table 4 Direct detection of Borrelia burgdorferi sensu lato in 43 skin biopsy specimens from classic acrodermatitis chronica atrophicans lesions using culture in BSK II medium, culture in MKP medium, and PCR amplification assay

\begin{tabular}{llll}
\hline BSK & $M K P$ & $P C R$ & Number \\
\hline+ & + & + & 3 \\
+ & + & - & 0 \\
+ & - & + & 1 \\
- & + & + & 1 \\
- & + & - & 6 \\
- & - & + & 2 \\
+ & - & - & 0 \\
- & - & - & 30 \\
\hline
\end{tabular}

specimens were positive by all three direct detection methods. This is shown in table 1, where the total numbers of specimens positive by each of the three methods alone, or in combination, are presented. Thus, only $11.5 \%(47$ of 408) of all positive specimens or $6 \%$ (47 of 758) of all specimens tested were positive by all three methods. Among positive specimens, the highest success rate was obtained by MKP culture alone $(272$ of $408(67 \%))$, followed by PCR assay alone (189 of $408(46 \%)$ ), followed by BSK II culture alone (179 of $408(44 \%)$ ). These differences were statistically significant $(p<<0.0001)$. The overall positivity rate for all three methods combined was $54 \%$ (408 of 758 ), for MKP culture $36 \%$ (272 of 758 ), for PCR 25\% (189 of 758), and for BSK II $24 \%$ (179 of 758). These differences were also statistically significant $(p<<0.0001)$.

The relative success rate of the three methods was not consistent throughout the two years of the study (fig 1). From November 1992 until September 1993, BSK II culture produced a consistently higher success rate than MKP culture. The success rate of PCR relative to BSK II fluctuated throughout this time period, sometimes being higher and sometimes lower. Beginning in October 1993, MKP culture was consistently more successful than either BSK II culture or PCR assay for the detection of spirochaetes; it was only surpassed by PCR during one month, August 1994. It was not possible to define clearly the reasons for this apparent reversal.

As some patients presented with multiple erythema migrans lesions, the total number of specimens tested exceeded the total number of patients (fig 1). For these patients also, individual lesions were rarely positive by all three methods, and the positivity of one lesion was not predictive of positivity in other lesions. This is illustrated in table 2 , where the results obtained from 14 representative patients are 

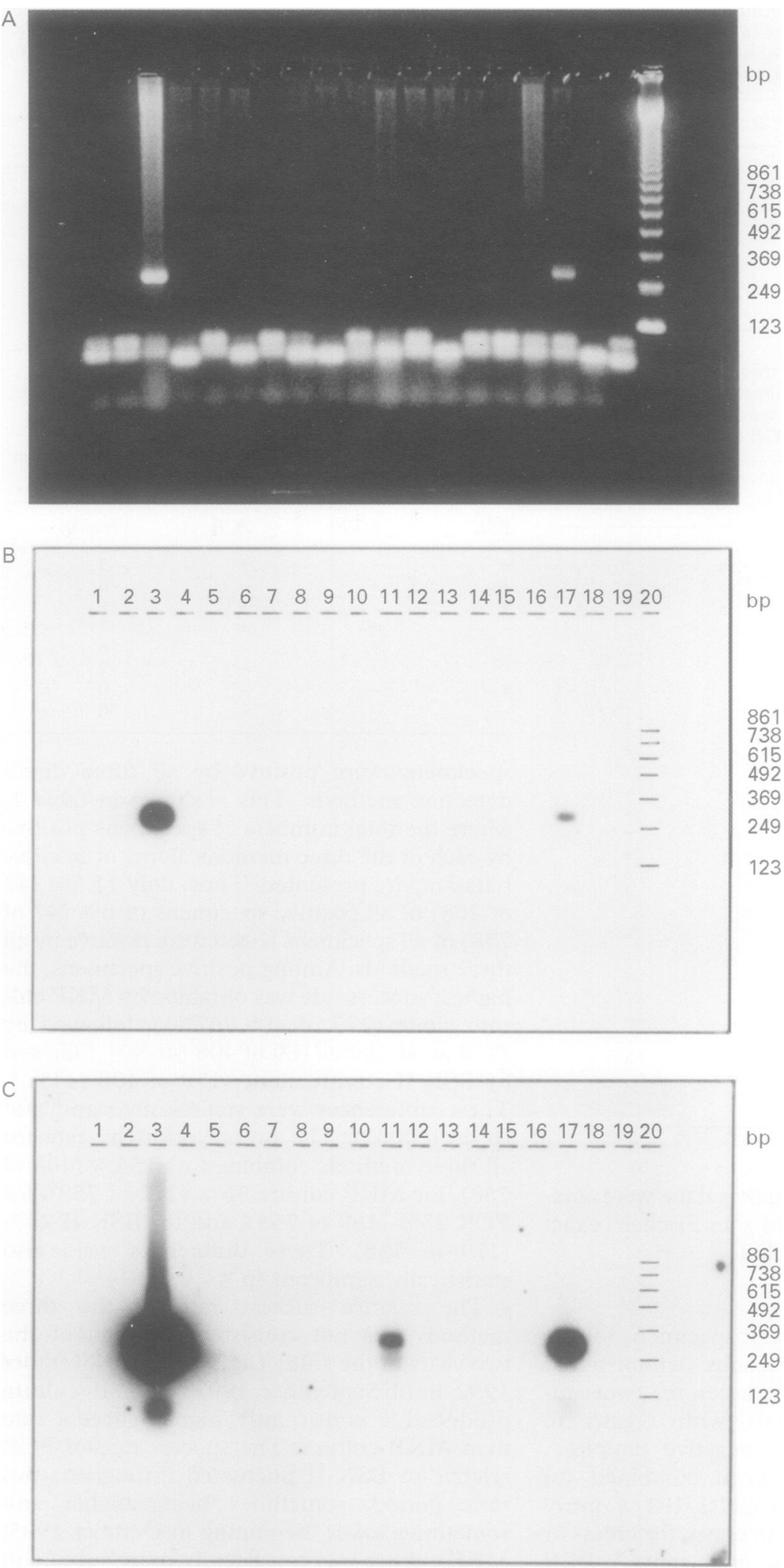

Figure 2 Polymerase chain reaction analysis $(A)$ of segments of skin biopsy specimens from 18 patients with typical erythema migrans lesions (lanes 1-18); lane 19, negative control specimen; lane 20, molecular size marker consisting of concatenates of a 123 base pair fragment. Agarose gel electrophoretic analysis of aliquots of the 19 samples after PCR amplification. Ethidium bromide staining revealed a visible amplification product of the correct size (276 base pairs) in the case of three specimens (3,11 (weak), and 17). Southern blot analysis $(B)$ of the agarose gel shown in $(A)$. The blot was probed with a 51 base pair probe (FL16 ${ }^{26}$ ) possessing sequence complementary to the central region of the 276 base pair amplification product. In this short autoradiographic exposure the two strongest bands seen in $(A)$ are seen to hybridise with the probe. A longer autoradiographic exposure $(C)$ of $(B)$. The bands produced in the case of specimens 3,11 , and 17 are seen to hybridise, indicating that they are specific amplification products. Specimen number 3 produced the strongest signal, indicating that it contained the most target sequences. Possible reasons for an additional minor amplification product of $\sim 105$ base pairs, consequent to an internal mis-priming event, have been discussed previously. ${ }^{27}$ presented. Comparison of positivity rates for MKP culture, BSK II culture, and the PCR amplification assay demonstrated significant differences per patient $(p=0.029)$ but not per sample $(p=0.083)$. However, examination of multiple lesions increased the overall diagnostic sensitivity. Of 38 patients from whom two erythema migrans lesions were biopsied and tested by all three detection methods, 28 (74\%) were positive for spirochaetes in at least one $\overline{0}$ lesion by at least one method.

In the case of a few patients, strong PCR sig- $\frac{\bar{m}}{7}$ nals indicative of the presence of large numbers $\stackrel{\mathbb{Q}}{2}$ of spirochaetes were obtained from one segment of a skin biopsy while adjacent segments $\overrightarrow{0}$ were culture negative. This is illustrated in fig 2 , where three patient specimens were seen to $\vec{\omega}$ produce a visible band after agarose gel $\stackrel{\circ}{3}$ electrophoresis (specimens 3, 11 (weak), and 17). After Southern transfer of the gel and $O$

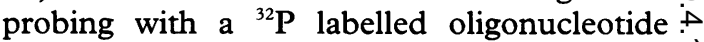
probe possessing an internal sequence comple- $\vec{\infty}$ mentarity to the amplification product, all three fragments hybridised (fig $2 \mathrm{~B}$ and $2 \mathrm{C}$ shows autoradiographs of this Southern blot $\gg$ exposed for successively longer periods of time) indicating that the amplification prod- की ucts were specific. However, whereas other skin $\vec{\bullet}$ segments from specimens 11 and 17 were positive by both MKP and BSK II culture, specimen number 3 (which produced the strongest PCR signal) was negative by BSK II culture. This result was obtained in November 1992 when BSK II culture outperformed MKP culture routinely in the detection of positive specimens.

ERYTHEMA MIGRANS STATUS POST

Skin appearing normal at the site of spontaneously resolved erythema migrans lesions was biopsied in a total of 120 patients. The results for all three detection methods are shown in table 3 . Only 10 biopsies were positive $(8 \%)$. Although the detection rate from erythema migrans status post lesions was very low, results showed the same random distribution of posi- $\frac{7}{0}$ tivity among the three detection methods as was found for typical erythema migrans $\sigma$ lesions. Differences in the positivity rate by $N$ MKP culture, BSK II culture, and PCR ampli- N fication assay were not statistically significant. 응

ATYPICAL/PARTIALLY TREATED ERYTHEMA MIGRANS LESIONS

Of 63 atypical erythema migrans lesions tested, one was positive both by MKP culture and $\underset{\mathbb{D}}{\circ}$ PCR. Thirty nine partially treated erythema migrans lesions also were tested. Of these, four $\cong$ were positive. One was positive by BSK II culture only, one was positive by MKP culture $\delta$ only, one was positive by PCR assay only, and the fourth was positive by both MKP culture and PCR. Differences in the positivity rate by MKP culture, BSK II culture, and PCR amplification assay were not statistically significant.

\section{CLASSIC ACRODERMATITIS CHRONICA} ATROPHICANS LESIONS

The results obtained from testing 43 skin biopsy specimens of classic acrodermatitis chronica 
atrophicans lesions are shown in table 4 . Thirteen specimens were positive overall, with MKP culture being most successful in detecting spirochaetes. However, differences were not statistically significant. Three specimens were positive by all three detection methods.

ATYPICALPARTIALLY TREATED ACRODERMATITIS CHRONICA ATROPHICANS LESIONS

Sixty six atypical acrodermatitis chronica atrophicans lesions were tested. Of these, one was positive by MKP culture only and one was positive by PCR only. Twenty lesions from patients who had received partial antibiotic therapy for acrodermatitis chronica atrophicans were tested; all were negative.

\section{Discussion}

From its inception, this study was not confined to a small, carefully selected group of patients displaying classic signs and symptoms of Lyme borreliosis. In addition to patients presenting with classic erythema migrans or acrodermatitis chronica atrophicans lesions and a history of tick bite, we also chose to investigate atypical lesions, lesions in patients who had received partial antibiotic treatment, and skin appearing to be clinically normal at the site of spontaneously resolved lesions (as reported by the patient). For the diagnosis of Lyme borreliosis, we do not consider that either clinical assessment, culture, or serological tests can serve as a "gold standard" against which assay by PCR can be evaluated and compared. Therefore, no attempt was made to calculate sensitivity and specificity on this basis. Also, it should be noted that the PCR and culture results reported here are based on a direct analysis of clinical specimens. We did not perform PCR analysis on partially-grown cultures of skin biopsies as reported previously. ${ }^{20}$ Although this procedure may enhance the sensitivity of the assay, it combines the slow turnaround time of culture with the high cost of PCR, both of which are undesirable in clinical diagnosis. To address the issue of sequence variability among European strains of $B$ burgdorferi sensu lato, primers derived from the highly-conserved flagellin gene were used for detection as discussed previously. ${ }^{21} 26$

Despite the wide range of skin biopsies tested, classic erythema migrans lesions constituted the vast majority of the specimens. This would be expected in a Lyme borreliosis endemic region. When total skin specimens per month were plotted, as in fig 1 , the result was essentially the same; the graph showed the same seasonal peaks and troughs but with total numbers that were slightly higher. The highest success rate in culturing borrelia from typical erythema migrans lesions was obtained on-site, using MKP medium, the aggregate rate over the two year period being $36 \%$. Culturing at a distance in BSK II medium was less successful $(24 \%)$. Reported culture isolation rates for borrelia from erythema migrans specimens have varied from a high of $86 \%{ }^{28}$ to less than $8 \%{ }^{29}$ However, the higher value represents results from a short term study of a small number of patient samples $(<30)$. Typically, culture isolation rates of $30-70 \%$ have been reported for erythema migrans specimens collected over longer time periods. ${ }^{30}{ }^{31}$ We found difficulty in attaining a consistently high isolation rate over the two year period of this study; as shown in fig 1 , success rates fluctuated over time. This was true also of direct detection by PCR assay, for which the overall success rate (25\%) was slightly higher than BSK II culture, but considerably lower than MKP culture. However, in certain months, the PCR assay outperformed both culture methods. We were not able to define precisely the reasons for the varying success rates of BSK II and MKP culture methods. No significant changes were made to the composition of either medium during the study period. However, variations in different batches of medium components (especially rabbit serum) may have played a role in this phenomenon.

Results for classic acrodermatitis chronica atrophicans lesions mirror those for classic erythema migrans, except that the overall numbers were smaller, and fewer specimens were positive. Thus, the acrodermatitis chronica atrophicans positivity rate for MKP culture was $23 \%$, that for the PCR assay was $16 \%$, and BSK II culture was $9 \%$. This was from a total of 43 specimens. Culture isolation rates from acrodermatitis chronica atrophicans lesions have typically been lower than from erythema migrans; however, in one report, a culture isolation rate of $45 \%$ from acrodermatitis chronica atrophicans was achieved. ${ }^{31}$ Among atypical acrodermatitis chronica atrophicans lesions, only two skin biopsies were positive from a total of 66 specimens tested.

A small number of erythema migrans status post specimens and skin biopsies from patients who had received partial antibiotic treatment also were positive by one or more methods. The isolation of spirochaetes from skin appearing normal at the site of spontaneously resolved erythema migrans lesions has been reported previously. ${ }^{23}{ }^{32}$ It could also be anticipated that in the case of incomplete antibiotic therapy, spirochaetes would still be present in the skin of some patients.

Throughout the study, many skin biopsy specimens were positive by only one, or two, of the three detection methods. Thus, by using more than one method, and more than one biopsy sample, the overall yield of positive results was increased. When all three methods were combined the overall positivity rate for typical erythema migrans lesions increased to $54 \%$ for analysis of a single lesion and $74 \%$ for two lesions. Tables 1-4 demonstrate an apparently random distribution of positive results among the three detection methods. This suggests that the distribution of spirochaetes within skin tissue at the site of an erythema migrans or acrodermatitis chronica atrophicans lesion is not uniform and that the chance of obtaining a positive result is subject to a sampling bias.

This is supported further by experimental PCR findings where one segment of an 
erythema migrans skin biopsy produced an exceptionally strong signal by PCR (detectable by agarose gel electrophoresis and ethidium bromide staining alone, without the use of a radioactively labelled probe), while other segments from the same skin biopsy were culture negative (fig 2). Agarose gel electrophoresis alone routinely detects $\sim 5000-10000$ spirochaetes after amplification by the conventional flagellin gene PCR assay used in this study. ${ }^{26}$ The very strong signal shown for specimen number 3 in fig $2 \mathrm{~A}$ easily represents the upper limit of this range. The results, therefore, seem to indicate that while one segment of a skin biopsy specimen may contain $10^{4}$ spirochaetes, adjacent segments may contain no spirochaetes, or an insufficient number to allow detection by culture.

It is conceivable that spirochaetes may be present in skin as microcolonies dispersed among regions that contain only a few scattered organisms. Neubert $e t a l^{3}$ investigated serial sections of erythema migrans and acrodermatitis chronica atrophicans skin lesions and found that, for most skin samples, not more than two spirochaetes were detectable in one or two out of 10 sections scanned. However, in the case of one skin biopsy, 10-15 spirochaetes were found in each of the $10 \mathrm{sec}-$ tions studied. Closely similar findings have also been reported by others. ${ }^{34}$ In the study by Neubert et $a{ }^{33}$ organisms were clustered around blood vessels deep in the dermis. More recently, they reported signs of small vessel vasculitis, consistent with a localised clustering of borrelia, in $38 \%$ of erythema migrans and $46 \%$ of acrodermatitis chronica atrophicans skin samples investigated using histological and/or immunohistological methods. ${ }^{35}$ Clusters of spirochaetes also have been observed in histochemically stained skin sections ${ }^{36}$ and synovial tissues. ${ }^{37}$

We conclude from these studies that in vitro amplification assays are not more sensitive than culture for the detection of borrelia in skin specimens and that the diagnosis of Lyme borreliosis by skin biopsy will remain problematical. Our findings indicate that the sensitivity of direct detection is limited and that the limitation occurs at the level of sample acquisition. While positive results by culture or PCR are highly specific, a negative result does not rule out Lyme borreliosis; however, examination of multiple specimens/lesions increases the likelihood of detecting spirochaetes. Although PCR has the potential to provide a rapid diagnosis, the necessarily complex laboratory logistics are costly, and the expenses involved in performing the assays themselves are not inconsiderable. In contrast, culture isolation, although slow in the case of some specimens, is relatively inexpensive and does not require a complex laboratory infrastructure. Moreover, the clinical value of a positive culture, as opposed to a positive PCR result, may be considered higher since in at least some patients PCR may detect dead spirochaetes not indicative of an active disease process.
This work was supported by grant AR 41517 from the National Institute of Arthritis and Musculoskeletal and Skin Diseases (to RNP), and by grants from the Microscan Division of Baxter Diagnostics (to RNP), and the Schweppe Foundation (to Jeffrey A Nelson in the screening of some BSK II cultures.

1 Stanek G, Satz N, Strle F, Wilske B. Epidemiology of Lyme borreliosis. In: Weber K, Burgdorfer W, Schierz G, eds. Aspects of Lyme borreliosis. Berlin: Springer-Verlag, 1993:358-70.

2 Ciesielski CA, Markowitz LE, Horsley R, Hightower AW, Russell H, Broome CV. The geographic distribution of Lyme disease in the United States. Ann New York Acad Sci 1988;539:283-8.

3 Burgdorfer W, Barbour AG, Hayes SF, Benach JL, Grunwaldt E, Davis JP. Lyme disease-a tick-borne spirochetosis? Science 1982;216:1317-19.

4 Postic D, Edlinger C, Richaud C, Grimont F, Dufresene Y, ڤ Perolat $\mathrm{P}$, et al. Two genomic species in Borrelia burgdorferi. Res Microbiol 1990;141:465-75.

5 Baranton G, Postic D, Saint Girons I, Boerlin P, Piffaretti JC, Assous M, et al. Delineation of Borrelia burgdorferi sensu stricto, Borrelia garinii sp. nov., and group VS461 associated with Lyme borreliosis. Int $\mathscr{f}$ Syst Bacteriol 1992; 42:378-83.

6 Marin Canica M, Nato F, du Merle L, Mazie JC, Baranton G, Postic D. Monoclonal antibodies for identification of Borrelia afzelii sp. nov. associated with late cutaneous manifestations of Lyme borreliosis. Scand F Infect Dis 1993; 25:441-8.

7 Åsbrink E, Hovmark A. Early and late cutaneous manifestations in Ixodes-borne borreliosis (erythema migrans borreliosis, Lyme borreliosis). Ann N Y Acad Sci 1988;539:4-15.

8 Steere AC. Lyme disease. N Engl f Med 1989;321:586-96.

9 Berger BW, Johnson RC, Kodner C, Coleman L. Failure of Borrelia burgdorferi to survive in the skin of patients with antibiotic-treated disease. $\mathcal{F}$ Am Acad Derm 1992;27:34-7.

10 Nadelman RB, Nowakowski J, Forseter G, Bittker S, Cooper D, Goldberg $\mathrm{N}$, et al. Failure to isolate Borrelia burgdorferi after antimicrobial therapy in culturedocumented Lyme borreliosis associated with erythema migrans-report of a prospective study. $A m$ f Med 1993;94:583-8.

11 Craft JE, Grodzicki RL, Steere AC. Antibody response in Lyme disease: evaluation of tests. $\mathcal{F}$ Infect Dis 1984;149: 789-95.

12 Berardi VE, Weeks KE, Steere AC. Serodiagnosis of early Lyme disease: analysis of IgM and IgG antibody responses by using an antibody-capture enzyme immunoassay. $\mathcal{f}$ by using an antibody-cap
Infect Dis 1988;158:754-60.

13 Magnarelli LA, Fikrig E, Berland R, Anderson JF, Flavell RA. Comparison of whole-cell antibodies and an antigenic flagellar epitope of Borrelia burgdorferi in serologic tests for diagnosis of Lyme borreliosis. $\mathcal{F}$ Clin Microbiol 1992;30: 3158-62.

14 Feder HM, Gerber MA, Luger SW, Ryan RW. Persistence of serum antibodies to Borrelia burgdorferi in patients treated for Lyme disease. Clin Infect Dis 1992;15:788-93.

15 Wallich R, Helmes C, Schaible UE, Lobet Y, Moter SE, Kramer MD, et al. Evaluation of genetic divergence among Borrelia burgdorferi isolates by use of OspA, fla, HSP60, and HSP70 gene probes. Infect Immun 1992;60:4856-66.

16 Dressler F, Whalen JA, Reinhardt BN, Steere AC. Western blotting in the serodiagnosis of Lyme disease. $\mathcal{F}$ Infect Dis 1993;167:392-400.

17 Barbour AG. Isolation and cultivation of Lyme disease spi- O rochetes. Yale F Biol Med 1984;57:521-5.

18 Preac-Mursic V, Wilske B, Schierz G. European Borrelia burgdorferi isolated from humans and ticks. Culture conditions and antibiotic susceptibility. Zbl Bakt Hyg A 1986;263:112-8.

19 Persing DH, Smith TF, Tenover FC, White TJ, eds. Diagnostic molecular microbiology. Washington, DC: American Society for Microbiology, 1993.

20 Moter SE, Hofmann H, Wallich R, Simon MM, Kramer MD. Detection of Borrelia burgdorferi sensu lato in lesional skin of patients with erythema migrans and acrodermatitis chronica atrophicans by ospA-specific PCR. f Clin Microbiol 1994;32:2980-8.

21 Picken RN. Polymerase chain reaction primers and probes derived from flagellin gene sequences for specific detection of the agents of Lyme disease and North American relapsing fever. $\mathcal{F}$ Clin Microbiol 1992;30:99-114.

22 Centers for Disease Control. Lyme disease surveillanceUnited States, 1989-1990. Morbid Mortal Weekly Rep 1991;40:417-21.

23 Strle F, Cheng Y, Cimperman J, Maraspin V, Lotric-Furlan S, Nelson JA, et al. Persistence of Borrelia burgdorferi sensu lato in resolved erythema migrans lesions. Clin Infect Di 1995;21:380-9.

24 Boom R, Sol CJA, Salimans MMM, Jansen CL, Wertheimvan Dillen PME, van der Noordaa J. Rapid and simple method for purification of nucleic acids. $\mathfrak{f}$ Clin Microbiol 1990;28:495-503.

25 Vogelstein B, Gillespie D. Preparative and analytical purification of DNA from agarose. Proc Natl Acad Sci USA 1979;76:615-17. 
26 Picken MM, Picken RN, Han D, Cheng Y, Strle F. Single-tube, nested polymerase chain reaction assay based on flagellin gene sequences for detection of Borrelia burgdorferi sensu lato. Eur $\mathcal{f}$ Clin Microbiol Infect Dis 1996;15:489-98.

27 Cheng Y, Han D, Picken RN, Picken MM, Nelson JA, Ruzic-Sabljic E, et al. Detection and diagnosis of Lyme borreliosis by culture and in vitro amplification assay. In: Cevenini R, Sambri V, La Placa M, eds. Advances in Lyme borreliosis research. Bologna: Societa Editrice Esculapio, 1994:171-4.

28 Berger BW, Johnson RC, Kodner C, Coleman L. Cultivation of Borrelia burgdorferi from erythema migrans lesions and perilesional skin. F Clin Microbiol 1992;30:350-61.

29 Hopkins M, Rutledge B, Persing DH. Culture of Borrelia burgdorferi for the diagnosis of Lyme disease: a three year experience. [abstract] 36th Interscience Conference on Antimicrobial Agents and Chemotherapy. New Orleans, LA, 1996:74.

30 Åsbrink E, Hovmark A. Successful cultivation of spirochete from skin lesions of patients with erythema chronicum migrans Afzelius and acrodermatitis chronica atrophicans. Acta Pathol Microbiol Scand (Section B) 1985;93:161-3.

31 Wilske B, Preac-Mursic V. Microbiological diagnosis of Lyme borreliosis. In: Weber K, Burgdorfer W, Schierz G, eds. Aspects of Lyme borreliosis. Berlin: Springer-Verlag, 1993:267-99.

32 Kuiper H, van Dam AP, Spanjaard L, de Jongh BM, Widjojokusumo A, Ramselaar TCP, et al. Isolation of Borrelia burgdorferi from biopsy specimens taken from healthylooking skin of patients with Lyme borreliosis. F Clin Microbiol 1994;32:715-20.

33 Neubert U, Krampitz HE, Engl H. Microbiological findings in erythema (chronicum) migrans and related disorders. Zbl Bakt Hyg A 1986;263:237-52.

34 Berger BW, Clemmensen OJ, Ackerman AB. Lyme disease is a spirochetosis. A review of the disease and evidence for its cause. Am F Dermatopathol 1983;5:111-24.

35 Neubert U, Kind P, Meurer M, Di Antonio S, Weber T, Volkenandt $M$. Evaluation of clinical features and laboratory data in erythema migrans and acrodermatitis chronica atrophicans patients [abstract]. VII International Congres on Lyme Borreliosis. San Francisco, CA, 1996:159.

36 Aberer E, Mainitz M, Neumann R, Stanek G. Immunoperoxidase staining of spirochetes in borrelial skin diseases. Ann N Y Acad Sci 1988;539:362-4

37 De Koning J, Hoogkamp-Korstanje JAA. Diagnosis of Lyme disease by demonstration of spirochetes in tissue biopsies. Zbl Bakt Hyg A 1986;263:179-88. 